\title{
Increased serum visfatin as a risk factor for atherosclerosis in patients with ischaemic cerebrovascular disease
}

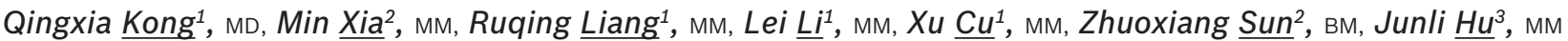

\begin{abstract}
INTRODUCTION The present study aimed to investigate the possible associations between serum levels of visfatin, an adipokine, and atherosclerosis in patients with ischaemic cerebrovascular disease.

METHODS A total of 95 participants were recruited for this study. Group A comprised 35 individuals with no history of cerebrovascular disease (control group) and Group B comprised 60 patients with ischaemic cerebrovascular disease. Group B was further categorised into two subgroups based on the ultrasonographic findings of the common carotid artery intima-media thickness (CCA-IMT) - Group B1 consisted of 21 patients with no atherosclerosis (i.e. CCA-IMT $\leq 0.9 \mathrm{~mm}$ ) and Group B2 consisted of 39 patients with atherosclerosis (i.e. CCA-IMT $>0.9 \mathrm{~mm}$ ). The body mass index, fasting blood total cholesterol, triglycerides, high-density lipoprotein cholesterol, low-density lipoprotein cholesterol and glucose levels of each patient were measured. Serum visfatin levels were determined using enzyme-linked immunosorbent assays. Visfatin levels were compared between groups, and stepwise logistic regression analysis was used to identify risk factors for atherosclerosis, including visfatin levels.

RESULTS The mean serum visfatin level of the patients in Group B was higher than that in Group A $(75.5 \pm 77.80 \mathrm{ng} / \mathrm{mL}$ vs. $8.6 \pm 4.69 \mathrm{ng} / \mathrm{mL} ; \mathrm{p}<0.05)$ and the level was higher in patients from Group B2 than those from Group B1 $(89.0 \pm 80.68 \mathrm{ng} / \mathrm{mL}$ vs. $50.4 \pm 72.44 \mathrm{ng} / \mathrm{mL} ; \mathrm{p}<0.05)$. Multivariate regression analysis showed that CCA-IMT values were not significantly associated with visfatin levels. However, logistic regression analysis showed that serum visfatin was an independent risk factor for atherosclerosis (odds ratio $37.80 ; p=0.004$ ).

CONCLUSION Serum visfatin may be an independent risk factor for cerebral infarction, as high serum visfatin levels are positively associated with the underlying pathogenic mechanisms of ischaemic cerebrovascular disease.
\end{abstract}

Keywords: adipocytokine, atherosclerosis, cerebral infarction, ischaemic cerebrovascular disease, visfatin

\section{INTRODUCTION}

Atherosclerosis is a form of chronic inflammation that results from the complex interplay among modified lipoproteins, elements of the innate (e.g. monocyte-derived macrophages) and acquired (e.g. T-cells) immune systems, and normal cellular elements of the arterial wall. ${ }^{(1)}$ Thus, in broad terms, the loss of local/systemic inflammation control, and aberrant energy and lipoprotein metabolism underlie the pathogenesis of atherosclerosis.

With regard to energy metabolism, adipose tissue is no longer considered just an energy storage repository. Due to its complex composition of multiple cell types (including leucocytes) and association with numerous normal metabolic and disease processes, adipose tissue is also considered an endocrine organ capable of producing hormone-like compounds called adipokines. $^{(2)}$ Adipokines (e.g. adiponectin, leptin, and resistin) are involved in important metabolic processes in vivo, playing significant roles in the regulation of glucose and lipid metabolism, and the development of insulin resistance (IR).

Adipose tissue also produces factors that can regulate inflammation, particularly vascular inflammation. ${ }^{(2)}$ Some of these adipokines appear to be involved in inflammation control (e.g. interleukin- 1 and tumour necrosis factor- $\alpha$ [TNF- $\alpha]$ ), while some provide cardiovascular protection (e.g. adiponectin and vascular endothelial growth factor) and others are distinctly proinflammatory (e.g. interleukin-8 and resistin). ${ }^{(2)}$ Their complex interactions have not yet been clearly delineated. However, with regard to atherosclerosis, proinflammatory adipokines have attracted considerable attention due to their possible association with known risk factors such as IR, obesity and aberrant lipoprotein metabolism.

Nicotinamide phosphoribosyltransferase (NAMPT) was first identified as a pre-B-cell colony-enhancing factor (PBEF) from a human peripheral blood lymphocyte cDNA library. ${ }^{(3)}$ It was subsequently found to have insulin-mimicking properties and designated visfatin due to its production/secretion in visceral fat tissue. ${ }^{(4)}$ Visfatin (also known as NAMPT or PBEF), is a pleiotropic protein that has at least three known specific functional properties: (a) it functions as an enzyme in the catalysis of the rate-limiting step of $\mathrm{NAD}^{+}$production from nicotinamide; (b) it is a novel insulin-mimetic fat-secreted factor; and (c) it is a regulatory factor in proinflammatory and immunomodulatory processes, which have been implicated in numerous disease processes, including atherosclerosis. ${ }^{(5-7)}$ In particular, visfatin appears to be a novel mediator of innate immunity; increased levels of visfatin were found in a number of chronic inflammatory diseases. ${ }^{(8)}$ Dahl et $\mathrm{al}^{(9)}$ found increased visfatin mRNA levels in macrophages associated with atherosclerotic plaques, suggesting that visfatin has a role in inflammation and plaque destabilisation. Visfatin has also been found to induce monocyte chemotactic protein-1 (MCP-1) secretion by human endothelial cells, suggesting a possible role in angiogenesis. ${ }^{(10)}$ Studies have found that increased serum visfatin levels were associated with atherosclerotic parameters 
in chronic haemodialysis patients ${ }^{(11)}$ and carotid atherosclerosis in patients with metabolic syndrome. ${ }^{(12)}$ Both studies emphasised the proinflammatory properties of visfatin.

With regard to cerebrovascular disease, Lu et al ${ }^{(13)}$ found that plasma visfatin levels were significantly increased in ischaemic stroke patients when compared to healthy controls. However, Lu et al did not examine the presence or the extent of atherosclerotic plaques, or their possible associations with visfatin levels. In this study, we sought to determine whether serum visfatin levels were associated with atherosclerosis in patients with ischaemic cerebrovascular disease (ICD). We also tested for possible associations between visfatin levels and other atherosclerosis risk factors, including body mass index (BMI), total cholesterol (TC) and low-density lipoprotein cholesterol (LDL-C).

\section{METHODS}

We recruited 60 patients (age range 40-70 years) who had had acute ischaemic cerebral infarctions and were seen as outpatients at the Affiliated Hospital of Jining Medical College, Shandong Province, China, from November 2009 to April 2010. The diagnosis of ischaemic cerebral infarction in these 60 patients was consistent with the diagnostic criteria established by the Fourth Nationwide Academic Conference on Cerebrovascular Disease of the Chinese Medical Association. ${ }^{(14)}$ Patients were excluded if they had cerebral haemorrhage, intracranial space-occupying lesion, acute cerebral embolism or lacunar infarction. Patients with a history of coronary artery disease or atrial fibrillation, any serious infectious disease, liver or kidney disease, any disease of the immune system, or malignant tumours were also excluded from the study. The selected patients were not on any lipid-lowering medication, anticoagulants or steroids.

We also recruited a control group of 35 healthy individuals (age range 30-60 years) who were seen in the outpatient clinics for routine physical examinations. These individuals did not have any history of acute cerebral infarction or any of the characteristics listed in the aforementioned exclusion criteria. This study was approved by the ethics committee of the Affiliated Hospital of Jining Medical College, and written informed consent was obtained from all the participants of this study.

The participants were allocated to Group A (controls) and Group B (patients who had acute cerebral infarction). Group B was further subdivided based on the patients' common carotid artery intima-media thickness (CCA-IMT), which was determined using the results of colour Doppler ultrasonography. Based on the 2007 European Society of Hypertension and European Society of Cardiology guidelines for standard hypertension treatment, ${ }^{(15)}$ the absence of atherogenesis is defined as CCA-IMT $<0.9 \mathrm{~mm}$, and patients from Group B who satisfied this criterion were categorised as Group B1. CCA-IMT $>0.9 \mathrm{~mm}$ indicates thickening (i.e. atherogenesis), and patients from Group B who satisfied this criterion were categorised as Group B2.

The height $(\mathrm{m})$ and weight $(\mathrm{kg})$ of each participant were measured, and the measurements obtained were used to calculate the BMI $\left(\mathrm{kg} / \mathrm{m}^{2}\right)$. Blood samples were obtained via sterile venipuncture after participants had fasted overnight. The blood samples were collected in sterile tubes with no anticoagulants. Serum was separated by routine procedures and sent to the hospital's clinical laboratory for measurement of TC, triglycerides (TG), high-density lipoprotein cholesterol (HDL-C), LDL-C and blood glucose levels. Measurements were made using an analyser (Hitachi 7600 Clinical Analyser; Hitachi High-Tech, Tokyo, Japan).

Serum visfatin levels were determined using an enzyme-linked immunosorbent assay (ELISA) kit, according to the supplier's instructions (Nampt/Visfatin/PBEF kit; Axxora Deutschland $\mathrm{GmbH}$, Lörrach, Germany). The results of the ELISA were read on an ASCENT ${ }^{\circledR}$ microplate reader (Thermo Fisher Scientific, Denver, CO, USA). This assay's sensitivity was $30 \mathrm{pg} / \mathrm{mL}$ and its range of detection was $0.125-8.0 \mathrm{ng} / \mathrm{mL}$. Serum samples were diluted prior to testing using a diluent provided with the ELISA kit. The normal human serum visfatin level is $15.8 \pm 16.7 \mathrm{ng} / \mathrm{mL} .{ }^{(16)}$

CCA-IMT was determined using standard procedures, ${ }^{(17)}$ with an Acuson ${ }^{\circledR}$ Doppler ultrasound system (Sequoia 512; Siemens, Munich, Germany). This procedure was performed by a single technician. Each participant was asked to lie in a prone position with the neck extended in a slightly lateral rotation. The carotid artery was scanned and the lumen, identified. The common carotid artery was checked transversally from the superior border of the internal extremity of the clavicle, and the probe was moved such that the cross section of the common carotid artery was positioned at the centre of the image. The probe was rotated close to $90^{\circ}$ to show the longitudinal section of the common carotid artery, and then moved toward the head to examine the topmost cervical part of the artery. Observing the common carotid artery lengthways, the artery's posterior wall would show two echo-level disjunctive parallel bright lines (i.e. a 'double-line' image). The intima-media thickness (IMT) is defined as the distance from the front edge of the first echogenic line (the lumen-intima interface) to the front edge of the second line (the media-adventitia interface). The IMT was measured three times on each side, and the average value of the measured values on both sides was recorded as the average CCA-IMT.

For continuous variables, data was presented as mean \pm standard deviation and Student's $t$-test for independent samples was used to compare data between the groups. Data for the categorical variable (i.e. gender) was presented as number and percentage, and categorical data between the groups were compared using chi-square test. Associations among variables were assessed using Spearman's rank correlation and multivariate linear regression analyses. Logistic regression analysis was used to determine whether increased serum visfatin was a risk factor for atherosclerosis. Statistical analysis was performed using the Statistical Package for the Social Sciences version 16.0 (SPSS Inc, Chicago, IL, USA). A p-value $<0.05$ was considered statistically significant.

\section{RESULTS}

Table I shows the demographic and clinical characteristics of the healthy controls (Group A; $n=35$ ) and patients who had 
Table I. Demographic and clinical characteristics of the study participants $(n=95)$.

\begin{tabular}{lrcc}
\hline Variable & \multicolumn{3}{c}{ Mean \pm SD } \\
\cline { 2 - 4 } & $\begin{array}{c}\text { Group A } \\
(\mathbf{n}=\mathbf{3 5})\end{array}$ & $\begin{array}{c}\text { Group B1 } \\
(\mathbf{n}=\mathbf{2 1})\end{array}$ & $\begin{array}{c}\text { Group B2 } \\
(\mathbf{n}=\mathbf{3 9})\end{array}$ \\
\hline Gender* & & & \\
Female & $11(31.4)$ & $7(33.3)$ & $11(28.2)$ \\
Male & $24(68.6)$ & $14(66.7)$ & $28(71.8)$ \\
Age (yrs) & $38.8 \pm 9.15$ & $55.5 \pm 10.53$ & $66.6 \pm 10.18$ \\
TC (mmol/L) & $4.7 \pm 1.03$ & $4.9 \pm 1.12$ & $5.1 \pm 0.87$ \\
TG (mmol/L) & $1.1 \pm 0.57$ & $1.8 \pm 0.99$ & $1.6 \pm 1.22$ \\
HDL-C (mmol/L) & $1.2 \pm 0.24$ & $1.0 \pm 0.32$ & $1.1 \pm 0.38$ \\
LDLC (mmol/L) & $2.7 \pm 0.74$ & $2.7 \pm 0.91$ & $2.9 \pm 0.65$ \\
Glucose (mmol/L) & $4.8 \pm 0.61$ & $6.2 \pm 3.01$ & $6.5 \pm 2.89$ \\
BMI (kg/m $)$ & $24.3 \pm 4.07$ & $26.2 \pm 2.77$ & $25.5 \pm 3.11$ \\
Visfatin (ng/ml) & $8.6 \pm 4.69$ & $50.4 \pm 72.43$ & $89.0 \pm 80.68$ \\
\hline
\end{tabular}

*Data is presented as no. (\%). Group A: patients with no history of acute cerebral infarction; Group B1: patients with a history of acute cerebral infarction and common carotid artery intima-media thickness (CCA-IMT) $\leq 0.9 \mathrm{~mm}$; Group B2: patients with a history of acute cerebral infarction and CCA-IMT $>0.9 \mathrm{~mm}$ BMI: body mass index; HDL-C: high-density lipoprotein cholesterol; LDL-C: low-density lipoprotein cholesterol; SD: standard deviation; TC: total cholesterol TG: triglycerides

had acute ischaemic cerebral infarction (Group B; $\mathrm{n}=60$ ). As previously described, Group B was subdivided into two groups: Group B1 $(n=21)$ consisted of patients without evidence of atherosclerosis (i.e. CCA-IMT $\leq 0.9 \mathrm{~mm})$; and Group B2 $(\mathrm{n}=39)$ consisted of those who showed evidence of atherosclerosis (i.e. CCA-IMT > $0.9 \mathrm{~mm}$ ).

Significant differences in age $(t=11.13 ; p<0.001)$, fasting TG levels $(t=3.15 ; p=0.002)$, fasting glucose levels $(t=4.13$; $p<0.001)$ and $\mathrm{BMI}(\mathrm{t}=1.99 ; \mathrm{p}=0.049)$ were found between Groups $A$ and $B$, with all of the aforementioned measures significantly higher in the latter. There were no significant differences between the two groups in terms of gender, fasting TC, fasting HDL-C and fasting LDL-C (all $p>0.05$ ).

Mean serum visfatin level of patients in Group B $(75.5 \pm 77.80 \mathrm{ng} / \mathrm{mL})$ was significantly higher than that in Group A $(8.6 \pm 4.69 \mathrm{ng} / \mathrm{mL})(\mathrm{t}=6.50 ; \mathrm{p}<0.001)$. Comparing the two subgroups in Group B, those who showed evidence of atherosclerosis (Group B2) had significantly higher visfatin levels than those without evidence of atherosclerosis (Group B1) $(t=3.57 ; p<0.001)$. These results suggest that increased serum visfatin levels are associated with the presence of atherosclerosis in our study population.

Using the combined data of Groups A and B, Spearman's rank correlation analysis was used to determine whether any significant associations exist between visfatin levels and other atherosclerosis risk factors. Significant positive correlations were detected between visfatin and two factors, namely TG $(p=0.027)$ and glucose $(p=0.004)$ levels. No significant correlations were found between visfatin and BMI, TC, HDL-C or LDL-C (all p > 0.05).

We also tested for possible associations between visfatin levels and other risk factors using multivariate regression analysis, with visfatin as the dependent variable. Due to the skewed distribution of visfatin, log-transformed values were used. No
Table II. Results of the multivariate regression analysis, with common carotid artery intima-media thickness as the dependent variable.

\begin{tabular}{lccc}
\hline Variable & Coefficient \pm SE & t-value & p-value \\
\hline Age & $0.44 \pm 0.00$ & 3.41 & 0.001 \\
TC & $-0.81 \pm 0.01$ & -2.85 & 0.006 \\
TG & $0.30 \pm 0.003$ & 1.91 & 0.061 \\
HDL-C & $0.25 \pm 0.012$ & 1.49 & 0.143 \\
LDL-C & $0.66 \pm 0.009$ & 2.47 & 0.017 \\
Glucose & $0.19 \pm 0.001$ & 1.69 & 0.097 \\
BMI & $-0.31 \pm 0.001$ & -2.74 & 0.008 \\
Log [visfatin] & $0.21 \pm 0.009$ & 1.80 & 0.078 \\
\hline
\end{tabular}

BMI: body mass index; HDL-C: high-density lipoprotein cholesterol; LDL-C: low-density lipoprotein cholesterol; SE: standard error; TC: total cholesterol; TG: triglycerides

significant associations between visfatin levels and BMI, TC, TG, HDL-C, LDL-C or glucose (all $p>0.05$ ) were found. That is, no systematic associations were observed between visfatin levels and other atherosclerosis risk factors in our study population.

For patients from Group B (i.e. both Groups B1 and B2), multivariate regression analysis was used to test for associations between CCA-IMT value (dependent variable) and the atherosclerosis risk factors assessed in this study. We found that CCA-IMT values were significantly associated with age $(p=0.001), T C(p=0.006)$, LDL-C $(p=0.017)$ and BMI $(p=0.008)$, but not significantly associated with TG, HDL-C, glucose, or (log-transformed) visfatin levels (all p > 0.05; Table II). This finding showed that visfatin levels were not associated with CCA-IMT.

We also used logistic regression analysis to determine whether increased visfatin levels in patients from Group B (i.e. both Groups B1 and B2) were a risk factor for atherosclerosis. The results of this analysis showed that (log-transformed) visfatin level was a risk factor $\left(\chi^{2}=8.515\right.$; odds ratio $\left.=37.797 ; p=0.004\right)$. As visfatin level was not associated with any of the other measured variables, increased visfatin levels appeared to be an independent risk factor for atherosclerosis development in our study population.

\section{DISCUSSION}

The present study demonstrated that serum visfatin levels were significantly increased in patients with ICD as compared to healthy individuals who have no history of ICD or other chronic inflammatory diseases. A similar finding was previously reported by Lu et al. ${ }^{(13)}$ The mean serum visfatin level of those in the control group (i.e. Group A) was $8.6 \pm 4.7 \mathrm{ng} / \mathrm{mL}$ (Table I), a value well within the normal range for healthy humans, which was previously reported by Chen et al to be $15.8 \pm 16.7 \mathrm{ng} / \mathrm{mL} .{ }^{(16)}$ Increased circulating levels of visfatin were also found to be associated with numerous chronic inflammatory diseases, such as inflammatory bowel disease, acute lung injury and rheumatoid arthritis. ${ }^{(5-7)}$

We also found that increased serum visfatin levels were associated with the occurrence of atherosclerosis in patients with ICD in our study (i.e. Group B), based on assessments of 
the CCA-IMT. Previous studies have also shown that increased visfatin levels were related to atherosclerosis in patients with cardiovascular disease, ${ }^{(5-7)}$ patients on chronic haemodialysis, ${ }^{(11)}$ and those with metabolic syndrome ${ }^{(12)}$ and spinal cord injuries. ${ }^{(18)}$ However, to the best of our knowledge, the present study is the first to show an association between increased circulating visfatin levels and atherosclerosis in patients with ICD.

Although it is likely that increased circulating visfatin plays a role in atherogenesis, it is unlikely that any single factor alone contributes to the pathogenesis of atherosclerosis. Atherosclerosis involves a complex interplay among modified lipoproteins, elements of the innate (e.g. monocyte-derived macrophages) and acquired (e.g. T-cells) immune systems, and normal cellular elements of the arterial wall. ${ }^{(1)}$ Furthermore, visfatin is a multifunctional protein; its three known functions are: (a) an enzyme in the catalysis of the rate-limiting step of $\mathrm{NAD}^{+}$production from nicotinamide; (b) a novel insulin-mimetic fat-secreted factor; and (c) a regulatory factor in proinflammatory and immunomodulating processes. ${ }^{(5-7)}$ Among these three functions, it is most likely that the formation of atherosclerotic plaques involves visfatin's proinflammatory properties. Although Lu et $\mathrm{al}^{(13)}$ did not investigate the occurrence of atherosclerosis in their group of patients with ICD, they did show that visfatin levels were positively correlated with high-sensitivity C-reactive protein (hs-CRP) levels. A similar association between visfatin and hs-CRP levels was also found in spinal cord injury patients with carotid arterial stiffness. ${ }^{(18)}$ Thus, it is possible that the mechanisms that promote a chronic inflammatory state also regulate and modulate visfatin production and activity.

Visfatin, in addition to having pleiotropic effects, is produced in numerous tissues and organs, including the adipose tissue, bone marrow, skeletal muscle and liver. ${ }^{(19)}$ It was first identified as a PBEF from a human peripheral blood lymphocyte cDNA library. ${ }^{(3)}$ With regard to inflammation, visfatin appears to be a novel mediator of innate immunity, as it can induce the production of inflammatory cytokines such as TNF- $\alpha$, interleukin- $1 \beta$ and interleukin-6. ${ }^{(8)}$ Dahl et al $^{(9)}$ found that visfatin expression was increased in macrophages found in human carotid artery plaques and associated with plaque destabilisation.

While visfatin's proinflammatory effects appear to promote atherogenesis, it remains unknown if these proinflammatory effects are independent of, or act in concert with, other proatherogenic mechanisms. For example, visfatin has been found to induce MCP-1 secretion by human endothelial cells, suggesting a role in angiogenesis. ${ }^{(10)}$ Visfatin's insulin-like activities could also play a pathogenic role through alterations in glucose metabolism. ${ }^{(4)}$ Visfatin has been shown to promote hypocellularity and mesentery proadipocytes in mice via an altered use of glucose and altered conversion of triglycerides, providing evidence that visfatin could affect lipid metabolism. ${ }^{(20)}$ It has also been found to interact with some mediators of inflammation (e.g. TNF- $\alpha$ and interleukin-8), resulting in increased endothelium permeability and smooth muscle cell proliferation. ${ }^{(21)}$ Visfatin can also inhibit nitric oxide synthase activity, leading to endothelium dysfunction. ${ }^{(22)}$ Thus, the proinflammatory effects of visfatin appear to be involved in the promotion of proatherogenic activities through changes in lipid metabolism and endothelial smooth muscle function.

Liu et $\mathrm{al}^{(23)}$ found that the level of circulating visfatin was associated with acute coronary artery disease, particularly acute coronary artery syndrome, and also reported that visfatin was an independent risk factor for coronary artery disease. In line with these results, the findings of the present study suggest that an increased circulating visfatin level is an independent risk factor for atherosclerosis development in patients with ICD.

In addition, we found that patient age, BMI, and blood TG, glucose and visfatin levels were all significantly increased in patients with ICD (i.e. Group B) as compared to the control group (i.e. Group A). It is generally accepted that age, BMI, TG level and glucose level are risk factors for cerebral infarction, and our results are consistent with this. Thus, increased serum visfatin levels may be a novel biomarker for atherogenesis in patients with ICD.

The results of our study also showed that CCA-IMT values correlated with age, TC, LDL-C and BMI. Multivariate regression analysis, however, revealed that serum visfatin levels were not associated with these variables (except for a possible association with age) or CCA-IMT values. Despite this, serum visfatin levels were found to be higher in patients with ICD and evidence of atherosclerosis (i.e. Group B2) than in patients with ICD and no evidence of atherosclerosis (i.e. Group B1). This suggests that increased serum visfatin levels are an independent risk factor for atherogenesis in patients with ICD.

The present study was not without limitations. As this was an exploratory study, we did not investigate other possible markers of inflammation, which may have provided clues regarding the pathway(s) involved in regulating visfatin or regulated by visfatin. Furthermore, while there was an apparent association between age and visfatin levels, the individuals in the control group (i.e. Group A) were significantly younger than the patients with ICD (i.e. Groups B1 and B2); this may have contributed to the observed difference in the visfatin levels. In particular, the mean age of patients with ICD in Group B2 (i.e. evidence of atherosclerosis present) was higher than that of patients with ICD in Group B1 (i.e. evidence of atherosclerosis absent). Thus, the association between age and visfatin level warrants further investigation. Finally, in a preliminary investigation using computed tomography brain images, we found evidence that the number and size of cerebral infarctions in Group B2 were greater than that in Group B1 (data not shown). While this could have been associated with age, it could also be due to increased circulating visfatin levels.

While visfatin is highly expressed in adipose tissue, especially stromal cells, it is also expressed in other tissues. ${ }^{(19)}$ Visfatin is differentially expressed in the adipose tissue of different organs, and this may influence its effects on atherogenesis. ${ }^{(20)}$ Although increased visfatin expression has been reported in diabetes mellitus, obesity, hypertension and cardiovascular disease, some reports suggest that visfatin levels may be low in these same diseases. ${ }^{(5-7)}$ There are multiple possible reasons for the discrepancies observed, such as differences in fat distribution, 
the presence or absence of inflammation, and changes in renal function, iron metabolism and hormone levels. ${ }^{(24)}$ Unravelling the interplay among these factors in order to aid understanding is a formidable challenge. Nonetheless, the results of the present study suggest that increased serum visfatin is an independent risk factor for the development of atherosclerosis in patients with ICD. Thus, the monitoring of visfatin levels and the development of drugs to inhibit visfatin may offer a means to prevent cerebral infarction.

\section{ACKNOWLEDGEMENTS}

We thank the instructors in the Statistics Department of Jining Medical College for their help with statistical analysis. We also thank Affiliated Hospital of Jining Medical College for match-funding this study. This study was approved and funded by the Health Department of Shandong Province HZ013 (2009).

\section{REFERENCES}

1. Glass CK, Witztum JL. Atherosclerosis: the road ahead. Cell 2001; 104:503-16.

2. Wozniak SE, Gee LL, Wachtel MS, Frezza EE. Adipose tissue: the new endocrine organ? A review article. Dig Dis Sci 2009; 54:1847-56.

3. Samal B, Sun Y, Stearns G, et al. Cloning and characterization of the cDNA encoding a novel human pre-B-cell colony-enhancing factor. Mol Cell Biol 1994; 14:1431-7.

4. Fukuhara A, Matsuda M, Nishizawa M, et al. Visfatin: a protein secreted by visceral fat that mimics the effects of insulin. Science 2005; 307:426-30.

5. Sommer G, Garten A, Petzold S, et al. Visfatin/PBEF/Nampt: structure, regulation and potential function of a novel adipokine. Clin Sci (Lond) 2008; 115:13-23.

6. Sonoli SS, Shivprasad S, Prasad CV, et al. Visfatin-a review. Eur Rev Med Pharmacol Sci 2011; 15:9-14.

7. Zhang LQ, Heruth DP, Ye SQ. Nicotinamide phosphoribosyltransferase in human diseases. J Bioanal Biomed 2011; 3:13-25.

8. Luk T, Malam Z, Marshall JC. Pre-B-cell colony-enhancing factor (PBEF)/ visfatin: a novel mediator of innate immunity. J Leukoc Biol 2008; 83:804-16

9. Dahl T, Yndestad A, Skjelland M, et al. Increased expression of visfatin in macrophages of human unstable carotid and coronary atherosclerosis. Possible role in inflammation and plaque destabilization. Circulation 2007; 115:972-80.
10. Adya R, Tan BK, Chen J, Randeva HS. Pre-B colony enhancing factor (PBEF)/visfatin induces secretion of MCP-1 in human endothelial cells: role in visfatin-induced angiogenesis. Atherosclerosis 2009; 205:113-9.

11. Kato A, Odamaki M, Ishida J, Hishida A. Relationship between serum pre-B cell colony-enhancing factor/visfatin and atherosclerotic parameters in chronic hemodialysis patients. Am J Nephrol 2009; 29:31-5.

12. Zhong M, Tan HW, Gong HP, et al. Increased serum visfatin in patients with metabolic syndrome and carotid atherosclerosis. Clin Endocrinol (Oxf) 2008; 69:878-84.

13. Lu LF, Yang SS, Wang CP, et al. Elevated visfatin/pre-B-cell colony-stimulating factor plasma concentration in ischemic stroke. J Stroke Cerebrovasc Dis 2009; 18:354-9.

14. Li W. The fourth cerebrovascular diseases academic meeting of the Chinese Medical Association: Diagnostic points of various cerebrovascular diseases. Chinese J Neurol 1996; 29:379-80.

15. Mancia G, DeBacker G, Dominiczak A, et al. 2007 Guidelines for the management of arterial hypertension: The task force for the management of arterial hypertension of the European Society of Hypertension (ESH) and of the European Society of Cardiology (ESC). Eur Heart J 2007; 28:1462-536.

16. Chen MP, Chung FM, Chang DM, et al. Elevated plasma levels of visfatin/ pre-B cell colony-enhancing factor in patients with type 2 diabetes mellitus. J Clin Endocrinol Metab 2006; 91:295-9.

17. Chien KL, Su TC, Jeng JS, et al. Carotid artery intima-media thickness, carotid plaque and coronary heart disease and stroke in Chinese. PLoS One 2008; 3:e3435.

18. La Favor JD, Hollis BC, Mokshagundam SL, Olive JL. Serum hsCRP and visfatin are elevated and correlate to arterial stiffness in spinal cord-injured subjects. Spinal Cord 2011; 49:961-6.

19. Sethi JK, Vidal-Puig A. Visfatin: the missing link between intra-abdominal obesity and diabetes? Trends Mol Med 2005; 11:344-7.

20. Spiroglou SG, Kostopoulos CG, Varakis JN, Papadaki HH. Adipokines in periaortic and epicardial adipose tissue: differential expression and relation to atherosclerosis. J Atheroscler Thromb 2010; 17:115-30.

21. Takebayashi K, Suetsugu M, Wakabayashi S, Aso Y, Inukai T. Association between plasma visfatin and vascular endothelial function in patients with type 2 diabetes mellitus. Metabolism 2007; 56:451-8.

22. Van derVeer E, Nong Z, O'NeilC, et al. Pre-B-cell colony-enhancing factor regulates $\mathrm{NAD}+$ dependent protein deacetylase activity and promotes vascular smooth muscle cell maturation. Circ Res 2005; 97:25-34.

23. Liu SW, Qiao SB, Yuan JS, Liu DQ. Association of plasma visfatin levels with inflammation, atherosclerosis and acute coronary syndromes (ACS) in humans. Clin Endocrinol (Oxf). 2009; 71:202-7.

24. Filippatos TD, Randeva HS, Derdemezis CS, Elisaf MS, Mikhailidis DP. Visfatin/PBEF and atherosclerosis-related diseases. Curr Vasc Pharmacol 2010; 8:12-28. 